\title{
Screening and Financial Contracting in the Face of Outside Competition
}

\author{
Dan Bernhardt and Stefan Krasa \\ Department of Economics \\ University of Illinois \\ Champaign IL 61820 \\ http://www.staff.uiuc.edu/ skrasa \\ danber@uiuc.edu
}

January 25, 2008

\begin{abstract}
This paper considers an investor who, at a cost, can acquire a signal about whether an entrepreneurial project will generate positive surplus. The problem for the potentially informed investor is that uninformed investors can compete to provide funding and the informed investor's contract offer conveys a signal to the entrepreneur about the project's likely payoffs, affecting the attractiveness to the entrepreneur of his uninformed funding alternatives. We determine how the investor's choices of signal quality and contracting terms are affected by project primitives. We prove that equilibrium expected payoffs in this signaling game are unique. We solve the optimal contracting problem for both the informed and uninformed investors, showing that informed investors can use equity, and that, under mild conditions, uninformed investors will compete with simple debt. Finally, we show that introducing the possibility of commitment by the informed investor alters the structure of the game, but not equilibrium payoffs.
\end{abstract}

Key words: screening, hidden information, entrepreneurial finance, outside competition, debt contracts, information acquisition 


\section{Introduction}

In the standard paradigms of the theory of contracting, monitoring by a principal serves two roles. Ex-ante monitoring is used to mitigate the impact of adverse selection: ex ante, the agent may have better information than the principal, and the principal's monitoring reduces any informational asymmetry. Ex-post monitoring is used to deter moral hazard by the agent, in particular to deter shirking or under-reporting of payoffs. Again, such monitoring mitigates moral hazard by reducing informational asymmetries.

While these are important aspects of contracting, this literature has largely overlooked how a principal's investigation can create information asymmetries that were not present previously. In particular, the principal may become better informed than the agent about some aspects of the project. This paper considers an investor who, upon a costly investigation, can become a better judge of the economic viability of an entrepreneur's project than the entrepreneur himself. The entrepreneur recognizes that while he may have a potentially-valuable invention, he may not be as good a judge of its market value: most entrepreneurs are associated with only a few projects, while experienced investors are exposed to a wide variety of projects. The investor's evaluation is important - most entrepreneurial projects go unfunded for good reason.

The entrepreneur understands that such an informed investor can serve two key roles. First, the investor investigates and filters projects. If the investor performs a sufficiently detailed investigation, then his willingness to fund the project represents a positive signal to the entrepreneur about the project's economic viability. Second, the informed investor can provide the capital necessary to fund the project. However, the informed investor is not the only party that can fund the project: post-investigation, the entrepreneur can obtain funding from an uninformed source.

The presence of this outside competition generates a signaling problem: the informed investor's contract conveys information to the entrepreneur about his screening. For example, if the informed investor does not offer a contract, this presumably conveys bad news to the entrepreneur. Indeed, if the screening is so accurate that the bad news signaled by a non-offer renders the entrepreneur unwilling to pursue the project, then outside investors can compete away any profits from projects that receive a good signal. This, in turn, means that the informed investor cannot cover his screening costs, and will therefore not screen the project. Accordingly, uninformed outside competition for funding induces the informed investor to distort the quality of information acquisition.

This paper develops the consequences of this outside funding competition for the equilibrium

screening and financial contracting choices of the informed investor. We consider a three-stage 
game. First, the investor chooses whether to initiate a costly investigation of the entrepreneur's project. If he investigates, the investor selects the quality of his signal. Next, if he investigates, the investor privately learns the signal realization, and decides whether or not to make a contract offer. A contract is a function $\kappa$ mapping project payoffs $x$ into the share $\kappa(x)$ of project payoffs that the investor receives. Simultaneously, an uninformed investor can offer a competing contract. Finally, the entrepreneur decides whether to accept a contract or to pursue his reservation alternative.

What is unusual about this signaling game is that in any subgame, the informed investor's equilibrium expected payoff is unique. Moreover, the equilibrium payoffs of the entire game to all parties are unique. We find that although mixing occurs in off-equilibrium subgames where too little information is acquired, on the equilibrium path there is no mixing. In fact, we show that on the equilibrium path, the contract and signal quality chosen by the informed investor solve a programming problem: maximize expected investor payoffs subject to inducing a lemons problem for uninformed investors. In equilibrium, the informed investor offers a contract with terms such that (1) following a good signal the entrepreneur is indifferent between acceptance and rejection if he believed that the informed investor's signal was bad with probability one; and (2) any investor who matches these terms and provides funds independently of the signal realizations, cannot expect a strictly positive payoff. Together these two conditions ensure that uninformed investors lose money if they undercut the equilibrium contract.

The surprising uniqueness of equilibrium is a consequence of inducing a lemons problem — given the most pessimistic beliefs possible about the signal, the entrepreneur would accept any contract that marginally undercuts the equilibrium contract. Hence, strange pessimistic beliefs cannot support multiple equilibria.

The equilibrium of the model also provides insight into how the informed and uninformed investors should design their contracts to affect the lemons problem: the informed investor would like to increase the severity of the lemons problem, while uninformed investor wants to mitigate it. Interestingly, it turns out that only the structure of the uninformed investor's contract matters, while the contract design by the informed investor does not affect the lemons problem. Thus, in practice, the contract design by the informed investor should be determined solely by the classical considerations of agency theory. In contrast, a target of contract design by the uninformed investor must always be to lessen the lemons problem. The uninformed investor does this by minimizing the attractiveness of his contract to the entrepreneur when the informed investor's contract signals that the project payoff is not high. If we allow for arbitrary contracts, then the uninformed investor's optimal contract is discontinuous, providing him the entire project in those states that a bad signal 
suggests are more likely, and providing zero payoffs, otherwise. However, in a setting with additional frictions, such contracts may generate incentive problems - for example, the investor would have incentives to interfere in the project to lower project payoffs, and the entrepreneur would have an incentive to report higher project payoffs (e.g., by augmenting payoffs with outside funds). This leads us to consider contracts where payments to each party are weakly increasing in the project payoff. We then show that the uninformed investor's unique optimal contract is simple debt as long as the distributions of project payoffs following good and bad signals are stochastically ordered by a simple dominance criterion.

Finally, we investigate how adding the possibility of commitment alters equilibrium outcomes. Not surprisingly, if the informed investor can commit ex ante to the signal quality and contracting terms, and the entrepreneur can commit to accepting the contract when offered, then the first best outcome is obtained. The informed investor extracts all informational rents, investigation quality is not distorted downwards, and investigation occurs if and only if it is socially desirable. What is more surprising is that if only the investor and not the entrepreneur can commit, then expected equilibrium payoffs are the same as in the game without commitment. The structure of the game is very different - there are many fewer subgames - but the investor must still generate a lemons problem, with the result that his ex ante contracting terms solve the same programing problem.

The paper is organized as follows. We next review the related literature. Section 2 introduces the model. To simplify the exposition and explicitly compute equilibria, Section 3 considers the special case where the project has only two possible realizations: success, $\bar{x}$, or failure, 0 . Then, contract design is trivial, as only the contract payoff following success, $\kappa(\bar{x}) \bar{x}$ matters. We explicitly characterize the properties of projects that are investigated, and solve for how the contracting terms, signal quality and investor profit vary with project primitives. Section 4 analyzes equilibria given a general state space for project payoffs. Section 5 shows how different levels of commitment by the different parties affect equilibrium outcomes. Section 6 discusses possible generalizations of the model. All proofs are in the Appendix.

\subsection{Related Literature}

Our paper contributes to three research areas: (a) competition between investors, (b) information acquisition and screening by investors, and (c) contract design and the optimality of simple debt contracts.

Broecker [3] exogenously endows investors (banks) with a signal about the entrepreneur's project, and details conditions under which the banks, who compete on interest rates, earn strictly 
positive profits. In contrast, we endogenize how the presence of an uninformed investor who can compete to provide funding affects the decision to become informed by a single investor, the quality of information that the investor acquires, and the form of contracting and contracting terms of both the informed and uninformed investors.

The distortion in information acquisition that we find has the flavor of the Grossman and Stiglitz [6] noisy rational expectations result. In their paper, if the equilibrium price is fully revealing then no information is acquired when information acquisition is costly. However, if, along the lines of Hellwig [7], there is added stochastic noise so that the competitive equilibrium price is partially revealing, then information may be acquired. In what follows we determine the endogenous amount of noise that arises in equilibrium when the investor chooses the signal quality and derive the characteristics of the economy for which endogenous noise supports costly information acquisition in equilibrium.

Using the language of principal-agent theory, we consider the information acquisition and contract design problem of a principal who seeks to design a contract that makes it worthwhile for him to acquire information. Cramer, Khalil and Rochet [8] consider the opposite problem for the principal of designing a contract that induces the agent to acquire information, building on the Baron and Myerson [2] model.

Our general contracting result that uninformed investors optimally employ simple debt contracts is novel. Standard explanations for simple debt contracts include costly state verification (Townsend [11], Gale and Hellwig [5], Williamson [13]) or costly enforcement (Krasa and Villamil [9]). Lacker and Weinberg [10] and Allen and Gale [1] show that non-contingent contracts do better at discouraging distortions in the reporting of profits.

We find that informed investors can use equity type contracts, while uninformed investors are determined in equilibrium to be simple debt contracts. This mirrors practice in the United States: venture capital and angel finance do not use debt, but banks, which typically employ only credit scoring as a screening device, use debt. Debt is optimal for uninformed investors because it minimizes adverse selection for them. Wang and Williamson [12] consider a different adverse selection problem caused by the presence of good and bad borrowers who know their types. They predict a separating equilibrium in which good and bad borrowers separate and types receive funding: in equilibrium, good borrowers self-select and choose debt funding, while bad borrowers are screened, and the structure of their contract is indeterminate. 


\section{The Model}

We consider an environment with three agents, a potential entrepreneur with a project and two potential investors $i=1,2$. All parties are risk neutral. The entrepreneur's project requires one unit of external funding in order to be developed. If developed, the project's payout is given by a random variable $X \geq 0$. The distribution of $X$ is common knowledge. If the entrepreneur does not take on the project, he can work for a reservation wage of $w>0$, which is public information. ${ }^{1}$

Investor 1 can acquire a signal about the project's payoff at a cost of $c>0$, while investor 2 is uninformed. This signal is private information to investor 1. To highlight investor 1's strategic incentives to limit information acquisition, we assume that up to some point, the marginal cost to investor 1 of a more accurate signal is zero. As a result, the only reason not to acquire better information is if superior information adversely affects the equilibrium financial contracting terms. There are two possible signal realizations: $\operatorname{good} g$, and bad $b .^{2}$ A higher signal quality $q \in[0,1]$ implies a higher expected payoff when the signal is good, and a lower expected payoff when the signal is bad. Formally, we assume that $E[X \mid \sigma(q)=g]$ is increasing in $q$ and that $E[X \mid \sigma(q)=b]$ is decreasing in $q$. Quality choice $q=0$ yields an uninformative signal, i.e., $X$ and $\sigma(0)$ are uncorrelated. Further, at $q=1$, a bad signal is sufficiently informative that $w>E[X \mid \sigma(1)=b]{ }^{3}$ Finally, we assume that the ex-ante probability of a good signal, $p=P(\sigma(q)=g)$, does not vary with $q$.

Investors can offer funding contracts $\kappa(\cdot)$ to the entrepreneur, where the payment to the investor is $\kappa(x) x$, and the payoff to the entrepreneur is $(1-\kappa(x)) x$. That is, without loss of generality, we focus on contracts that specify the state-contingent share of the realization that each party receives.

We allow for the possibility that the space $\mathfrak{S}$ of potential contracts may be restricted, e.g., by continuity, differentiability or monotonicity requirements. However, unless otherwise stated, we only impose the following restrictions on $\mathfrak{S}$ :

Measurability: All $\kappa \in \mathfrak{S}$ are measurable.

Closedness: Let $\kappa_{n} \in \mathfrak{S}$ for all $n \in \mathbb{N}$ and let $\lim _{n \rightarrow \infty} \kappa_{n}=\kappa$ a.e. Then $\kappa \in \mathfrak{S}$.

\footnotetext{
${ }^{1}$ This outside wage alternative also captures the decision problem for potential key employees of a start-up, workers with significant alternative opportunities. An alternative interpretation of $w$ is that it represents a utility cost of working for the firm, for which the entrepreneur must be compensated.

${ }^{2}$ The unique equilibrium that we characterize also exists when there are more than two signals. In particular, there always exists an equilibrium in which there is pooling on the same funding terms following all sufficiently good signals, and pooling on non-funding on all sufficiently bad signals. See the conclusion for a further discussion.

${ }^{3}$ This assumption is sufficient but stronger than necessary for our results to hold. This stronger assumption eases exposition.
} 
Convexity: $\mathfrak{S}$ is convex.

Limited Liability: $\kappa(x) \in[0,1]$ for a.e. $x \in \mathbb{R}_{+}$and for all $\kappa \in \mathfrak{S}$.

Fixed Share: $\mathfrak{S}$ contains the functions $k \equiv 0$ and $k \equiv 1$.

With convexity, the fixed share restriction implies that, at the very least, $\mathfrak{S}$ contains the set of all fixed equity contracts, which we denote by $k \in[0,1]$. For most of our analysis, we impose no other structure on the set $\mathfrak{S}$.

The game proceeds as follows:

Stage 1 Investor 1 chooses whether or not to spend funds $c$ to investigate the entrepreneur. If he investigates, he chooses a signal quality, $q$ that is public information. ${ }^{4}$

State 2 If he investigates, investor 1 privately observes the signal realization. Investors $i=1,2$ simultaneously choose whether or not to make a contract offer. If investor $i$ makes an offer, then he selects $\kappa_{i} \in \mathfrak{S}$.

Stage 3 If a financing contract is offered, the entrepreneur decides whether to pursue the project, or to take his outside wage option, $w$. If the entrepreneur pursues the project, he selects his preferred financing contract.

If funding by investor $i$ is accepted, then investor $i$ 's project payoff gross of any costs of investigation is $\kappa(x) x-1$, while the entrepreneur's payoff is $(1-\kappa(x)) x$, where $x$ is the project realization. If the entrepreneur rejects all contracts then the gross project payoff is zero. The informed investor's net payoff consists of the gross project payoff minus any investigation costs.

\section{Two States}

In this section we assume that the only possible realizations of $X$ are 0 and $\bar{x}$. This structure facilitates analysis as the universe of contracts $\mathfrak{S}$ is reduced to a non state-contingent share $k_{i}$ that investor $i$ receives when the realization $\bar{x}$ occurs.

\subsection{Becoming Well Informed Can be Unprofitable}

A key theme of our paper is that competition leads to low, socially inefficient, levels of information acquisition. To highlight this point and to provide intuition for subsequent results, we consider the

\footnotetext{
${ }^{4}$ For example, this assumption is natural in the case of venture capital finance, where the duration of the investigation is strongly correlated with the quality. In particular, the entrepreneur sees the thoroughness of the investigation.
} 
special case where investor 1 can only acquire one quality of informative signal, $\bar{q}$. If information is acquired, and the signal, $\sigma(\bar{q})=b$, then project payoffs are not expected to cover the entrepreneur's costs, i.e., $E[X \mid \sigma(\bar{q})=b]<w$ (i.e., the bad signal realization is very informative).

Theorem 1 Suppose that there is only one signal quality $\bar{q}$ and that $E[X \mid \sigma(\bar{q})=b]<w$. Then in any Perfect Bayesian Nash equilibrium, information is not acquired and investors' expected profits are zero.

If investor 1 becomes informed, then the entrepreneur values financing only if he receives a contract offer from the informed investor. This follows from the condition that $E[X \mid \sigma(\bar{q})=b]<w$. In particular, let investor 1 offer share $k_{1}$. Then $E\left[\left(1-k_{1}\right) X \mid \sigma(\bar{q})=b\right] \leq E[X \mid \sigma(\bar{q})=b]<w$.

If the informed investor does not offer a contract, then the entrepreneur can infer that the signal must have been bad, in which case he would prefer his reservation wage alternative. But then uninformed investor 2 knows that his contract offer would be accepted only if the signal is good. As a consequence, when the signal is good, Bertrand competition again reduces profits gross of the information acquisition cost down to zero. It follows that information acquisition is unprofitable. The proof extends this reasoning to preclude the possibility of profitable mixed strategies.

Theorem 1 has the flavor of the Grossman and Stiglitz (1980) noisy rational expectations result. There, if the equilibrium price is fully revealing then no information is acquired if information acquisition is costly. However, if, along the lines of Hellwig (1980), there is added stochastic noise so the competitive equilibrium price is partially revealing, then information may be acquired. In what follows we determine the endogenous amount of noise that arises in equilibrium when the informed investor chooses the signal quality. In particular, we determine the characteristics of the economy for which endogenous noise supports costly information acquisition in equilibrium.

\subsection{Equilibria with Information Acquisition}

We now return to the original model in which investor 1 can acquire any signal quality $q \in[0,1]$. Investor 1's dilemma is that he wants a more accurate signal in order to reduce the probability of financing a bad project. However, if he lowers this probability by too much, uninformed investor 2 can profitably undercut any profitable offer by the investor 1 .

We begin with a lemma that characterizes all equilibria of stage 2 subgames.

Lemma 1 There exist subgame perfect equilibria in all subgames starting at stage 2 for any given q. The expected equilibrium subgame payoff to informed investor 1 is unique. 
Let $k(q)$ solve $E[(1-k(q)) X \mid \sigma(q)=b]=w$, if such a $k(q)$ exists. Let $\underline{k}$ solve $E[\underline{k} X]=1$ if such $a \underline{k}$ exists.

1. If $E[X \mid \sigma(q)=g]-1 \leq w$, then the expected gross payoff to investor 1 is zero and no investment takes places.

2. If $E[X \mid \sigma(q)=g]>1+w$ and $E[X \mid \sigma(q)=b]<w$, then investor 1's gross payoff is zero.

3. If $E[X \mid \sigma(q)=g]>1+w$ and $E[X \mid \sigma(q)=b] \geq w$ then $k(q)$ exists.

(a) If $E[k(q) X] \leq 1$, and $E[k(q) X \mid \sigma(q)=g]>1$ then in the equilibrium subgame investor 1's expected gross profit is 0 if $\sigma(q)=b$ and $E[k(q) X \mid \sigma(q)=g]-1$, if $\sigma(q)=g$. The following is an equilibrium of the subgame: Investor 1 offers $k_{1}=k(q)$ if and only if $\sigma(q)=g$; uninformed investor 2 offers $k_{2}=k(q)$ with sufficiently high probability, and he never makes an offer $k_{2}<k(q)$. Investor 1's contract is accepted by the entrepreneur.

(b) If $E[k(q) X]>1$, and $E[k(q) X \mid \sigma(q)=g]>1$, then there exists a unique mixed strategy equilibrium. In this subgame investor 1's expected gross profit is $E[\underline{k} X \mid \sigma(q)=g]-1$ when $\sigma(q)=g$ and 0 , otherwise.

(c) If $E[k(q) X \mid \sigma(q)=g] \leq 1$, then investor 1's gross profits are zero.

The game with information acquisition is a signaling game, one where informed investor 1's offer of $k_{1}$ can reveal information about his type - his signal about whether the project is good. The surprising and unusual result in Lemma 1 is that in any subgame with information acquisition, the payoff to the informed investor is unique in equilibrium, without any appeal to refinements save for subgame perfection. Typically, dynamic games of incomplete information support many different equilibrium payoffs absent strong equilibrium refinements.

The key equilibrium determinant is the share value $k(q)$ at which the entrepreneur is indifferent between accepting and rejecting an offer if he knows for sure that $\sigma(q)=b$. For investor 1 to obtain strictly positive gross profits, the share $k_{1}$ cannot exceed $k(q)$. If it did, investor 2 could undercut $k_{1}$ without fear of being accepted when investor 1 does not make an offer, i.e., when $\sigma(q)=b$. But then Bertrand competition would immediately lower gross profits to zero. Thus, to obtain strictly positive gross profits that would allow investor 1 to cover his information acquisition costs, share $k_{1}$ cannot exceed $k(q)$.

In signaling games, multiple equilibria are typically supported by beliefs that a particular action choice by an informed agent reveals that his type is bad. For example, in an education signaling game, a firm may believe that a student's type is bad unless he acquires an excessive level of 
education, thereby supporting a continuum of equilibria with different payoffs. This logic does not apply to our game. In particular, suppose there were an equilibrium in which the investor 1 offered a contract $k_{1}<k(q)$ that is accepted by the entrepreneur, and there is no competing offer by investor 2. In standard signaling games, such strategies can be supported as equilibrium by beliefs that assign the bad type to any offer $k_{1}^{\prime}>k_{1}$ together with beliefs that the type is good when $k_{1}^{\prime} \leq k_{1}$. But here even if the entrepreneur believes with probability one that investor 1 observed a bad signal following $k_{1}^{\prime}$ with $k_{1}<k_{1}^{\prime}<k(q)$, the entrepreneur is still strictly better off accepting the contract, as $E\left[(1-k(q) X \mid \sigma(q)=b]=w<E\left[\left(1-k_{1}^{\prime}\right) X \mid \sigma(q)=b\right]\right.$. Therefore, optimization by investor 1 then leads him to share $k(q)$.

Theorem 2 proves that any equilibrium with information acquisition solves the following optimization problem:

\section{Problem 1}

$$
\max _{q, k_{1} \in[0,1]} p\left(E\left[k_{1} X \mid \sigma(q)=g\right]-1\right)-c
$$

subject to

1. $E\left[\left(1-k_{1}\right) X \mid \sigma(q)=b\right]=w$.

2. $E\left[k_{1} X\right] \leq 1$.

The objective in Problem 1 is investor 1's ex-ante expected profit given that finance is extended if and only if the project viability signal is good, i.e. if and only if $\sigma(q)=g$. Investor 1 chooses the project signal quality $q$ and equity share $k_{1}$ to maximize these profits.

To understand why constraints 1 and 2 must hold in equilibrium, first note that if information acquisition is too accurate, i.e., if $q$ is too close to one, then constraint 1 is violated as $E\left[\left(1-k_{1}\right) X \mid \sigma(1)=b\right]<w$. The left-hand side of constraint 1 is the entrepreneur's expected payoff when investor 1 receives a bad signal. The right-hand side is the entrepreneur's payoff when he does not pursue the project. Constraint 2 ensures that an uninformed investor who undercuts investor 1 's terms, and hence in view of constraint 1 funds the project in all states of the world, cannot profit.

\section{Theorem 2}

1. If investor 1 acquires information, then investor 1's equilibrium share $k_{1}$ and signal quality $q$ solve Problem 1 and $E\left[k_{1} X \mid \sigma(q)=g\right]-1-c \geq 0$, i.e., investor 1's payoff is non-negative. 
2. Conversely, let $k_{1}$ and $q$ solve Problem 1 and suppose that $E\left[k_{1} X \mid \sigma(q)=g\right]-1-c \geq 0$. Then there exists an equilibrium where investor 1 acquires information, and selects share $k_{1}$ and signal quality $q$.

\subsection{Explicit Characterizations and Comparative Statics}

In this two signal setting, it is without loss of generality to assume that the probability of a good signal is $p$ and that a signal of quality $q$ yields the true state with probability $q$, but is a random draw from the prior with probability $1-q$, where $q \in[0, \bar{q}]$. One can solve Problem ?? explicitly for the equilibrium values of $q$ and $k_{1}$ as a function of $p, w$ and $\bar{x}$. Figure 1 illustrates how investor 1's equilibrium expected payoff varies with the probability $p$ that the project is viable (when $\bar{q}=1$ ).

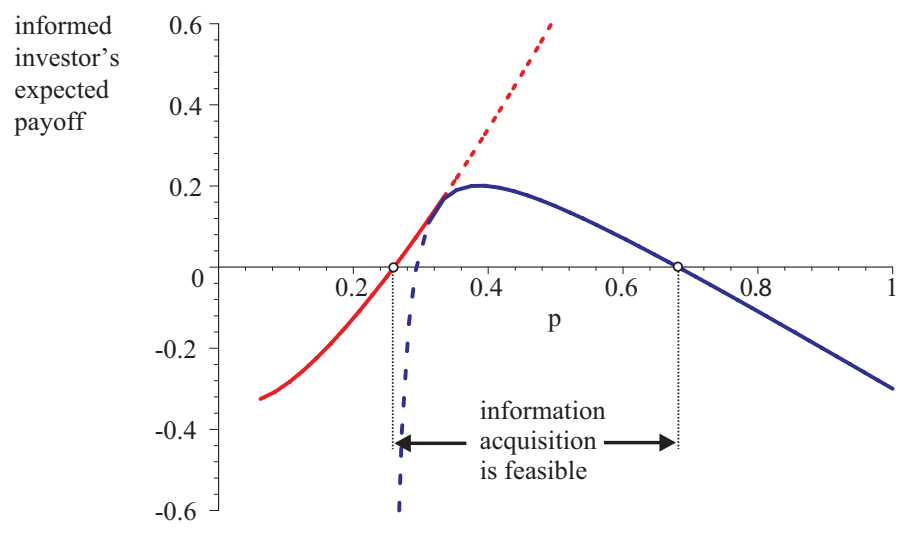

Figure 1: The profit of an informed investor

The graph shows that informed investor's ex-ante expected profit for the following parameter values: $\bar{x}=4, w=0.1, c=0.3 . p$ is the probability that $X=\bar{x}$

One can show that the key features illustrated in Figure 1 hold for arbitrary $w$ and $\bar{x}$. In particular, as Figure 1 illustrates, if $p$ is too small or too large, then informed finance is infeasible. This is because when $p$ is small, investor 1 cannot even find a $k_{1}$ that induces a lemons problem for the uninformed investor; and when $p$ is large, uninformed investor 2 would face little risk from competing if investor 1 tried to retain enough surplus to cover $c .^{5}$ Thus, equilibria with information acquisition only exist for intermediate values of $p$.

Also, as in Figure 1, for small values of $p$ where only constraint 1 binds, profits are a convex, increasing function of $p .{ }^{6}$ In this region, $E\left[k_{1} X\right]<1$ so that investor 2 would lose money by offering

\footnotetext{
${ }^{5}$ Note that for such safe projects both constraints of Problem 1 bind when $\bar{x}>1$. Combining the constraints reveals that $E[X]-1>w$ (i.e., the project's surplus covers $w$ ). As a result the project receives investment in equilibrium, but no information is acquired.

${ }^{6}$ In the figure the solid line is the actual payoff. The dotted portion of the convex curve shows investor 1 's profit were we to ignore the fact that constraint 2 binds, so that $E\left[k_{1} X\right]>1$. The dotted part of the concave curve assumes
} 
$k_{2}=k_{1}$. Therefore, it is sufficient for investor 1 to generate a lemons problem $-k_{1}$ and $q$ are not limited in any other way. The intuition for the convexity is as follows. When the project is unlikely to pay off, it is hard to make investor 2's contract attractive to the entrepreneur when $\sigma(q)=b$ investor 1 must both take a small share of the firm and choose a low signal quality. Fixing $k_{1}$ and $q$, investor 1's ex-ante payoff would increase linearly in $p$, as the objective (1) would be the product of $p$ and a constant. But, in addition, raising $p$ makes it is easier to induce a lemons problem. As a result, investor 1 can increase both $q$ and $k_{1}$ without inviting uninformed competition. This increase of $k_{1}$ and $q$ raises ex-ante payoffs from linear in $p$ to convex.

Investor 1's ex-ante payoff function becomes concave in $p$ when constraint 2 binds so that $E[k X]=1$. Once constraint 2 binds (at the tangency point of the convex and concave curves), when $p$ is raised further, investor 1 must lower $k$ to prevent investor 2 from undercutting his contract, i.e., to retain $E\left[k_{1} X\right]=1$. Therefore, the linear increase in ex-ante payoff due to an increase in $p$ is dampened by the reduction of $k_{1}$, causing payoffs to be concave in $p$. Finally, because the slope of ex-ante payoffs is positive at the tangency point, it follows that the $p$ that maximizes investor 1's ex-ante payoff is on the concave portion.

Figure 1 suggests that investor 1 prefers riskier projects. Still, we cannot yet draw this conclusion as $p$ affects both a project's mean return and its risk. We now show that the value of information acquisition is greater if, ceteris paribus, the project's variance is higher. It follows that a risk-neutral informed investor prefers riskier projects, and that projects with sufficiently large surplus, $E[X]-1>w$, that are too safe can only receive uninformed investment.

Theorem 3 For a given expected project payoff p $\bar{x}$, increasing the project variance strictly raises investor 1's ex-ante expected profit whenever screening occurs in equilibrium. Informed investment is not feasible if the project's variance is too low.

\section{General State Space for Project Payoffs}

We now consider the general setting that allows for many project realizations. With many possible project realizations, if we simply restrict attention to equity contracts the analysis in the previous section (e.g., Lemma 1) extends immediately. However, we want to allow for a broader class of contracts than just equity in order to address how investors design contracts in response to the screening problem, and how this feeds back to affect equilibrium outcomes.

that constraint 2 binds, so that $E\left[k_{1} X\right]=1$, even when $p$ is small enough that investor 1 does better by choosing a smaller share and acquiring better information. 
If the random variable $X$ describing project payoffs has a finite support then we do not need to impose additional structure on its distribution. If $X$ is a continuous random variable, then we assume that the distribution of $X$ conditional on signal $\sigma(q)=s, s=b, g$ has a density function with respect to the Lebesgue measure. Problem 2 below is the generalization of Problem 1 to the multi-state environment.

\section{Problem 2}

$$
\max _{q \in[0,1], k, \kappa^{U}} p(E[k X \mid \sigma(q)=g]-1)-c
$$

subject to

1. $E\left[\left(1-\kappa^{U}(X)\right) X \mid \sigma(q)=b\right]=w$.

2. $E\left[\kappa^{U}(X) X\right] \leq 1$,

where $\kappa^{U}(\cdot)$ solves Problem 3:

$$
\begin{aligned}
& \min _{\tilde{\kappa}^{U} \in \mathfrak{S}} E\left[\left(1-\tilde{\kappa}^{U}(X)\right) X \mid \sigma(q)=b\right] \\
& \text { s.t. } E\left[\tilde{\kappa}^{U}(X) X \mid \sigma(q)=g\right]=E[k X \mid \sigma(q)=g] .
\end{aligned}
$$

The objective in Problem 2 reveals that investor 1 can continue to use a pure equity contract. However, uninformed investor 2 wants to design his contract to minimize the attractiveness of his contract to the entrepreneur when $\sigma(q)=b$. Hence, his contract solves Problem (3). Constraints 1 and 2 of Problem 2 are the analogues of the constraints of Problem 1. First, offering any contract that is more attractive than $\kappa^{U}$ results in this contract being accepted even when $\sigma(q)=b$. The second constraint implies that investor 2 would lose money from doing so.

Before we state the our general equilibrium characterization result, we provide a lemma proving that solutions to optimization problem (3) exist and that the objective is continuous and strictly decreasing in $k$.

Lemma 2 Indexing investor 2's optimal contract by the share $k$ offered by investor 1, Problem 3 has a solution $\kappa_{k}^{U}(x)$ for all $k \in[0,1]$. Moreover, $E\left[\left(1-\kappa_{k}^{U}(X)\right) X \mid \sigma(q)=b\right]$ is a continuous, strictly decreasing function of $k$.

Lemma 2 ensures that if $E[X \mid \sigma(q)=b]>w$, then there exists a $\kappa$ that solves problem (3) such that $E[(1-\kappa(X)) X) \mid \sigma(q)=b]=w$. Define $k(q)$ to be the fixed share that gives the entrepreneur the same payoff as $\kappa$. Then this $k(q)$ plays the same role as the contract with share $k$ that fulfills 
$E[(1-k) X) \mid \sigma(q)=b]=w$ in the analysis of Problem 1. In particular, suppose that investor 1 offers a contract with fixed share $k(q)$ (or any other contract that gives the entrepreneur the same payoff). Then investor 2 faces a lemons problem if he undercuts: if investor 2 offers any contract $\hat{\kappa}$ with a higher payoff to the entrepreneur than $k(q)$ when $\sigma(q)=b$, then this contract will always be accepted in the bad state as $E[(1-\hat{\kappa}(X)) X) \mid \sigma(q)=b]>w$.

We are now ready to state our equilibrium characterization result for general state spaces: We pose the theorem in the context of a project that needs to be investigated to receive funding. The project could have a positive ex ante NPV, $E[X]-1>0$, but the surplus is not sufficiently large to cover the entrepreneur's alternative opportunity, $w$. In practice, $w$ may be high if the potential entrepreneur has significant human capital and hence has a high reservation alternative, e.g., a good salary at an existing job. A project with $E[X]-1>w$ could, in principle, be funded without investigation. In practice, if, for example, we consider venture capital finance, only $10-15 \%$ of projects that receive extensive scrutiny by a $\mathrm{VC}$ are funded by the $\mathrm{VC}$, indicating that the ex ante surplus from these projects is unlikely to cover $w$.

Theorem 4 Suppose that the project's ex-ante expected payoff is less than the reservation wage of the entrepreneur, i.e., $E[X]-1<w$. Then

1. If investor 1 acquires information and $q^{*}$ and $k^{*}(\cdot)$ are the equilibrium investigation intensity and contract choice, then $k=\frac{E\left[k(X) X \mid \sigma\left(q^{*}\right)=g\right]}{E\left[X \mid \sigma\left(q^{*}\right)=g\right]}$ and $q=q^{*}$ solve Problem 2 and $E\left[k^{*} X \mid \sigma\left(q^{*}\right)=\right.$ $g]-1-c \geq 0$, i.e., investor 1's payoff is non-negative. Uninformed investor 2 offers a $\kappa^{U}(\cdot)$ that solves Problem (3).

2. Conversely, let $k^{*}$ and $q^{*}$ solve Problem 2. Let $\kappa^{U}(\cdot)$ solve Problem 3 and suppose that $E\left[k^{*} X \mid \sigma\left(q^{*}\right)=g\right]-1-c \geq 0$. Then there exists an equilibrium where investor 1 selects a non-state contingent share $k^{*}$, investigation intensity $q^{*}$, and funds the project if and only if $\sigma\left(q^{*}\right)=g$. Uninformed investor 2 always offers $\kappa^{U}(\cdot)$.

In the proof of Theorem 4 we first show that because $E[X]-1<w$, if informed investor 1 offers a contract following a bad signal, he must hide this from the entrepreneur by offering this same contract with sufficient probability after the good signal, else the entrepreneur would have to lose money (as investor 1 would not offer an unprofitable contract). That is, investor 1 cannot always offer a contract when $\sigma(q)=b$, else the entrepreneur's expected payoff cannot be raised to $w$. However, such randomization by the informed investor implies that his expected profits from a contract that is accepted when $\sigma(q)=b$ must be zero. This observation simplifies the analysis of the game because it means that we can focus on investor 1's expected profits when $\sigma(q)=g$. 
The proof of Theorem 4 follows that of Theorem 2. The main difference is that investor 2 does not offer a contract with a fixed share, but rather a contract $\kappa(x)$ that solves Problem 3 . Investor 2 does this to minimize his exposure to lemons. In sharp contrast, the particulars of informed investor 1's contract do not matter. Indeed, suppose there are two contracts, $\kappa^{A}$ and $\kappa^{B}$ that are not equal to $\kappa^{U}$ but are payoff equivalent in the good state. Then, if $\kappa^{A}$ is accepted in any equilibrium subgame, so is $\kappa^{B}$. Again, the reason is that even if the entrepreneur has the most pessimistic belief that the signal is bad, it pays him more than $w$ and hence he strictly prefers to accept it. That is, the particular design of investor 1's contract are not affected by the information acquisition/signaling problem - the only way to affect the lemons problem is via the signal quality $q$ and the expected contract payoff following a good signal. This means that in practice, other considerations, such as classical agency issues, will guide investor 1's contract design.

Our assumptions on $X$ guarantee existence of equilibria for all stage 2 subgames. Existence of solutions to Problem 2 follows immediately when $X$ has only finitely many realizations. If $X$ is continuous, our existence argument uses the assumption that the distribution of $X$ contingent on signal $s=g, b$ changes continuously in $q$.

\subsection{Characterization of Investor 2's Contract Design}

If there are no other constraints on the set of feasible contracts $\mathfrak{S}$ that investor 2 can offer, then it is easy to show that given a project payoff $x$, investor 2's optimal contract either pays the entire project payoff to the entrepreneur or to investor 2 . In particular, letting $f_{j}(x)$ be the distribution over $x$ following $\sigma(q)=j, j=b, g$, one can partition $X$ into according to the relative likelihood, $\frac{f_{g}(x)}{f_{g}(x)+f_{b}(x)}$, of the good signal conditional on the realization: the entrepreneur receives the entire project payoff when $x$ is such that the relative likelihood of the good signal was high enough, and investor 2 receives the entire project payoff when the relative likelihood of the good signal was lower-where the boundary of the partition is determined by the constraint $E\left[\tilde{\kappa}^{U}(X) X \mid \sigma(q)=g\right]=E[k X \mid \sigma(q)=g]$.

However, there are many reasons to believe that the set of feasible contracts is smaller, due to incentive compatibility issues that arise in practice. In particular, if either the investor's payoff or the entrepreneur's payoff is not weakly increasing in $x$, then significant moral hazard concerns emerge. For example, if the investor's share is decreasing in $x$ on some range, then the investor has an incentive to shirk on supplying inputs or verifying cash flows, and to take actions (e.g., excessive monitoring) that lower output; while the entrepreneur has an incentive to mix in cash flows from other sources, so that he gets the entire payoff (and his cash injection is returned).

These considerations lead us to explore investor 2's equilibrium contract design when we add a 
monotonicity restriction to the set of feasible contracts: $\kappa^{U} \in \mathfrak{S}$ only if $x^{\prime}>x$ implies $\kappa^{U}\left(x^{\prime}\right) x^{\prime} \geq$ $\kappa^{U}(x) x$. We next show that with this monotonicity restriction on the set of feasible contracts that under a modest stochastic ordering restriction, investor 2's optimal contract is simple debt. In particular, we assume that the conditional likelihood, $\frac{f_{g}(x)}{f_{g}(x)+f_{b}(x)}$, of the good signal is increasing in the realization, $x$, i.e.,

$$
\frac{f_{g}^{\prime}(x)}{f_{g}(x)}>\frac{f_{b}^{\prime}(x)}{f_{b}(x)}
$$

in the interior of the support. Most standard densities satisfy this condition. For example, with the exponential distribution, $f^{\prime}(x) / f(x)=-1 / \lambda$, so that (4) holds if $f_{g}$ has a higher mean $\lambda$ than $f_{b}$. So, too, for a lognormal distribution, $f^{\prime}(x) / f(x)=\frac{-\zeta^{2}-\ln (x)+\lambda}{\zeta^{2} x}$, where $\lambda$ is the $\log$ of the median, and $\zeta$ is the standard deviation of $E[\ln (X)]$, so that if $f_{g}$ has a higher median, keeping $\zeta$ fixed, then (4) holds.

Theorem 5 Suppose that $\mathfrak{S}$ only contains contracts $\kappa$ for which payments to the investor, $\kappa(x) x$ are non-decreasing, and that (4) holds. Then, uninformed investor 2 uses a simple debt contract.

The intuition for the result is simple. To minimize the lemons problem, investor 2 wants to give more to the entrepreneur following realizations that are associated with higher likelihoods of the good signal, which under (4) are the higher states. The investor's ability to do this is constrained by the monotonicity restriction that his own payoff cannot decline in those high states - a flat payment is the best that he can do, i.e., simple debt is optimal. ${ }^{7}$

\section{$5 \quad$ Ex-ante Contracting}

\subsection{Full Commitment}

Given the distortion in information acquisition that emerge in all equilibria of our model, the question arises as to whether the distortion can be reduced or eliminated by ex-ante contracting. Ex-ante contracting requires commitment by the entrepreneur and the investor to the terms of the contract. For example, such commitment may be supported for the investor by reputation concerns.

The game with full commitment proceeds as follows:

\footnotetext{
${ }^{7}$ One can derive the optimality of simple debt, weakening the assumption on the distributions of project realizations following good and bad signals to the standard ordered hazard condition, $\frac{f_{b}(x)}{\left(1-F_{b}(x)\right)}>\frac{f_{g}(x)}{\left(1-F_{g}(x)\right)}$, for $x$ in the interior of the support of $F_{b}$ (which implies $F_{b}(x \mid x>\bar{x}) \geq F_{g}(x \mid x>\bar{x})$ for any $\bar{x}$ ); but adding a monotonicity requirement on the share that the entrepreneur receives, $\left(1-\kappa\left(x^{\prime}\right)\right) x^{\prime} \geq(1-\kappa(x)) x$. The dual monotonicity condition implies that $\kappa(x) x$ is continuous in $x$.
} 
1. Investor 1 can offer a contract specifying a signal quality $q$ and share $k_{1}$ that applies if he offers funding.

2. The entrepreneur decides whether to accept the contract.

(a) If the entrepreneur accepts the contract, investor 1 investigates and offers signal-contingent funding.

(b) If the entrepreneur rejects the contract, the game without commitment detailed in section 2 starts.

Theorem 6 The equilibrium of the game with full commitment has the following features:

1. There is no distortion in signal acquisition, i.e., $q=1$, and no commitment to $q$ is necessary.

2. The equilibrium payoff to the entrepreneur is the same as without ex-ante contracting. Investor 1 extracts all of the additional surplus from the higher quality investigation.

At the stage where the entrepreneur chooses whether to accept the ex-ante contract it follows immediately that he will do so if and only if his expected payoff from accepting is at least as high as his payoff from the original game, which is the solution to Problem 2 as shown in Theorem 4. Given our assumption that projects are unfundable following a perfectly accurate bad signal, i.e., $E[X \mid \sigma(1)=b]<w$, it follows immediately that $q=1$ violates the first constraint of Problem 2 , and hence $q<1$ in equilibrium. Thus, with full commitment investor 1 can increase total surplus, maximizing it by setting $q=1$. Given the entrepreneur's strategy it follows immediately that investor 1 extracts all surplus. Also, since it is optimal to set $q=1$, there is no need to write $q$ into the contract.

\subsection{Partial Commitment}

Commitment by the informed investor to a particular contract can be motivated by reputational concerns. It is far harder, however, to justify commitment by the entrepreneur. In practice, an entrepreneur cannot be forced to take funding and start a project against his will. In particular, once $q=1$ has been chosen and a contract offered, the entrepreneur may not accept that all surplus goes to investor 1 and could bargain for better terms. The entrepreneur's ability to do this hinges on his outside option, i.e., on what he can get from an alternative investor. We therefore amend the game with full commitment to allow the entrepreneur to reject investor 1's initial offer ex-post and pursue finance from investor 2. The game is as follows: 
1. Investor 1 can offer a contract specifying a signal quality $q$ and a contract $\kappa_{s}^{1}(\cdot)$ that applies if he extends funding, where the contract can be contingent on the announced signal $s=g, b$.

2. If the entrepreneur accepts the ex-ante contract, then

(a) Investor 1 investigates and offers signal-contingent funding.

(b) Investor 2 simultaneously offers $\kappa^{2}(\cdot)$ (equivalently, investor 2 does not see whether investor 1 offers funding).

(c) The entrepreneur chooses a contract from one of the investors or rejects both of them.

3. If the entrepreneur rejects the ex-ante contract, the game without commitment detailed in section 2 starts.

Theorem 7 In the game with partial commitment the expected equilibrium payoffs to all agents are unique. Payoffs correspond to those in the game with no commitment of section 2.

Equilibria to the game with partial commitment are quite different from the game without commitment. In particular, there is no mixing in subgames, and investor 2 need not make offers. This latter result follows because investor 1, when choosing his contract, anticipates the possibility of an offer from investor 2 if he does not design his contract appropriately.

On first contemplation, the different structures of the equilibria make it surprising that commitment by investor 1 cannot improve equilibrium outcomes. But reflection resolves this puzzle - both with partial commitment and without, investor 1 must create a lemons problem for investor 2; and Theorem 2 demonstrates that in the game without commitment, investor 1 chooses the signal quality and contracting terms that maximize his expected payoff subject to the constraint of inducing a lemons problem. It then follows immediately that investor 1 does no better with commitment.

\section{Conclusion}

Our paper characterizes how potential competition from uninformed investors affects the quality of screening and the structure of optimal contracts. We characterize how the presence of outside competition to provide funding distorts information acquisition, and prove that the optimal contract offered by uninformed investors is simple debt. Remarkably for a signaling framework, we find that equilibrium payoffs are unique, and we characterize how payoffs vary with the primitives of the economy. 
One simplification of our otherwise general model is that we assume that the informed investor receives only a binary signal. With more than two signals, the "two-signal" equilibrium that we characterize continues to be a natural equilibrium of the more general setting. In particular, in this equilibrium, the uninformed investor uses a simple debt contract, and there is pooling by the informed investor across states: the informed investor offers the same "best" contract if he offers funding, and no contract in the remaining states. Thus, the entrepreneur updates in the same way as in the two-signal model. The pooling by the informed investor on his "best" contract when he makes an acceptable offer is robust in senses that can be made precise. However, this is not true when the informed investor does not offer an acceptable contract: ex post, the informed investor is indifferent between the unacceptable contracts that he offers when he does not provide funding, and such offers can reveal information to the entrepreneur that could affect his willingness to pursue uninformed investment. We have not investigated the welfare properties of these equilibria, and suspect that they are ambiguous.

Our other simplifying assumption is that the entrepreneur's reservation utility, $w$, is public information at the time that contracts are offered. One could imagine that $w$ is private information to the entrepreneur - both investors only know the distribution over $w$. When the distribution over $w$ is continuous, the pure strategy equilibrium that we describe ceases to exist. Rather, the unique equilibrium structure mirrors the mixed strategy equilibrium that we identify in subgames (see the proofs of Lemma 1 or Theorem 4) where the informed investor acquires too little information. ${ }^{8}$ In the equilibrium, the informed investor's mixed strategy gives the uninformed investor zero expected profits - the informed investor induces a lemons problem in expectation. The uninformed investor does not always compete, but when he does he uses a simple debt contract to minimize the lemons problem. When the uninformed investor does offer the debt contract, he sometimes manages to undercut profitably the informed investor's contract when there is a good signal; but at the same time the uninformed investor faces a lemons problem and loses money when the informed investor receives a bad signal and the entrepreneur's realization of $w$ is too low.

\footnotetext{
${ }^{8}$ The construction of the contracting portion of this equilibrium is also similar to that in Engelbrecht-Wiggans, Milgrom, and Weber [4], who consider a sealed-bid auction with one informed bidder and competing uninformed bidders.
} 


\section{Appendix}

Proof of Theorem 1. We first prove that in any subgame starting at stage 2, informed investor 1 cannot make strictly positive gross (of investigation costs) profits. Suppose that information is acquired in equilibrium. Then $E[X \mid \sigma(\bar{q})=b]<w$ implies that $E\left[\left(1-k_{1}\right) X \mid \sigma(\bar{q})=b\right] \leq E[X \mid \sigma(\bar{q})=$ $b]<w$. Thus, the entrepreneur will never accept a contract if he knows that $\sigma(\bar{q})=b$.

Let $k_{l, g}^{I}$ be the lower support of investor 1's possibly mixed equilibrium contract offer and suppose by way of contradiction that $E\left[k_{l, g}^{I} X \mid \sigma(\bar{q})=g\right]-1>0$. This, however, implies that investor 2 must have strictly positive expected payoffs in equilibrium, because he could make money by undercutting $k_{l, g}^{I}$ marginally. This implies that both investors must make strictly positive gross profits at the upper supports $k_{h, g}^{I}$ and $k_{h}^{U}$ of equilibrium contract offers of the informed and uninformed investor, respectively. This would only be possible if $k_{h, g}^{I}=k_{h}^{U}$ and both are played with strictly positive probability. This is an immediate contradiction, because either party could strictly improve by reducing marginally the upper support of their equilibrium contract offer: the marginally smaller offer discontinuously raises the probability of acceptance at an infinitesimally small share reduction. Thus, $E\left[k_{l, g}^{I} X \mid \sigma(\bar{q})=g\right]-1 \leq 0$. Because investigation costs $c>0$, it follows that it is not optimal for investor 1 to acquire information.

Proof of Lemma 1. Case 1. There does not exist a $k$ that gives an investor and the entrepreneur non-negative expected payoffs. Hence, no mutually acceptable offer exists, so that all equilibria in this subgame involve no investment and payoffs are zero.

Case 2. To construct an equilibrium, choose the $k$ that solves $E[k X \mid \sigma(q)=g]=1$. Investor 1 offers $k_{1}=k$ if and only if $\sigma(q)=g$, and investor 2 always offers $k_{2}=k$. The entrepreneur accepts an offer if and only if investor 1 makes an offer. To see that this is an equilibrium, observe first that investors earn zero expected profits. A unilateral increase in $k_{1}$ or $k_{2}$ yields zero profits as it is always rejected, and a decrease is accepted whenever investor 1 makes an offer and results in negative expected profits. Finally, the signal is revealed through investor 1's action and because $E[X \mid \sigma(q)=b]<w$, the entrepreneur rejects investor 2's offer whenever investor 1 does not make an offer.

Now suppose by way of contradiction that there exists an equilibrium in which investor 1 makes strictly positive gross profits. Let $k_{h, g}^{I}$ be the highest offer in the support of investor 1's possibly mixed strategy on the equilibrium path when $\sigma(q)=g$. This offer must be accepted with strictly positive probability, else profits would be zero. If investor 1 makes an offer when $\sigma(q)=b$, then 
denote the highest such offer accepted on the equilibrium path by $k_{h, b}^{I}$. Then $k_{h, b}^{I} \leq k_{h, g}^{I}$, else the entrepreneur learns that $\sigma(q)=b$ and $E[X \mid \sigma(q)=b]<w$ implies that he would reject any such offer.

Suppose that $k_{h, b}^{I}<k_{h, g}^{I}$. Then the offer of $k_{h, g}^{I}$ reveals that $\sigma(q)=g$. Thus, if investor 2 offers $k_{h, g}^{I}-\varepsilon$, he makes strictly positive profits for $\varepsilon>0$, sufficiently small: (i) the offer is accepted with strictly positive probability since it is in the relative interior of the support of investor 1's mixed strategy; and (ii) the offer is only accepted by the entrepreneur if there is a higher offer by investor 1 , as such an offer reveals $\sigma(q)=g$. Thus, investor 2's expected equilibrium profits must be strictly positive. Let $k_{h}^{U}$ be the highest offer in the support of investor 2's possibly mixed strategy. Thus, $k_{h}^{U}$ must also be accepted with strictly positive probability, else investor 2's expected profits would be zero. This is only possible if $k_{h, g}^{I}=k_{h}^{U}$ and both are played with strictly positive probability. But, this is a contradiction, as investor 2 could profitably deviate by marginally lowering the upper end point of his support.

Finally suppose that $k_{h, b}^{I}=k_{h, g}^{I}$. This offer is accepted with positive probability. Thus, $E\left[k_{h, b}^{I} X \mid \sigma(q)=b\right] \geq 1$. Hence, investor 2 can also make positive profits, and a contradiction as above obtains.

Case 3a. We first argue that the strategies described in the statement of the Lemma are an equilibrium. In equilibrium, uninformed investor 2's payoff is zero. If investor 2 offers $k_{2}<k(q)$, then $E\left[\left(1-k_{2}\right) X \mid \sigma(q)=b\right]>w$ so that the offer is always accepted. Thus, investor 2's profit would be $E\left[k_{2} X\right]-1<0$. If investor 2 offers $k_{2}>k(q)$ then his offer is never accepted and profits remain zero. Informed investor 1 would obviously lower his payoff if he offers $k_{1}<k(q)$. If he offers $k_{1}>k(q)$ then investor 2's offer of $k_{2}=k(q)$ will be accepted if any offers are accepted, and investor 1's payoff becomes zero.

We next characterize all equilibria of the subgame.

First, note that any offer $k<k(q)$ is acceptable to the entrepreneur independently of his beliefs. In particular, $k<k(q)$ implies $E[(1-k) X \mid \sigma(q)=b]>w$, so that such an offer is attractive to the entrepreneur even if he believes that $\sigma(q)=b$. Next, investor 1 never makes an offer $k_{1}<k(q)$ that is accepted with positive probability when $\sigma(q)=b$. Since $E[k(q) X] \leq 1$ it follows that $E\left[k_{1} X \mid \sigma(q)=b\right]-1<0$ for $k_{1}<k(q)$, and hence investor 1 would lose money.

Let $k_{l}^{U}$ be the minimum of the support of investor 2's equilibrium mixed strategy. Then $k_{l}^{U} \geq$ $k(q)$. Otherwise, if $k_{l}^{U}<k(q)$ then $E\left[\left(1-k_{l}^{U}\right) X \mid \sigma(q)=b\right]>w$ and the offer is attractive to the entrepreneur even if he believes that $\sigma(q)=b$. This will be accepted when $\sigma(q)=b$ with probability 1 because, as shown above, investor 1 does not make offers $k_{1}<k(q)$. Since $k_{l}^{U}<k(q)$ 
and $E[k(q) X]<1$ it follows that investor 2 loses money, a contradiction.

Next, note that investor 1's gross expected payoff is strictly positive when $\sigma(q)=g$. In particular, suppose investor 1 offers $k_{1}$ that is marginally smaller than $k(q)$. Since $k_{l}^{U} \geq k(q)$ investor 1 's offer beats the offer by investor 2 , and since $k_{1}<k(q)$ the offer will be accepted. Since $k_{1}$ is only marginally smaller than $k(q)$ and $E[k(q) X \mid \sigma(q)=g]>1$ it follows that investor 1's equilibrium expected profits are strictly positive.

We now prove that $k_{h, g}^{I}=k_{l, g}^{I}=k(q)$, i.e., investor 1 offers $k(q)$ with probability 1 . Since $k_{l}^{U} \geq k(q)$ it follows immediately that $k_{l, g}^{I} \geq k(q)$; otherwise, investor 1 could strictly increase profits by increasing $k_{l, g}^{I}$, as the offer would be accepted with probability 1 . Now suppose by way of contradiction $k_{h, g}^{I}>k(q)$. We now show that investor 2's equilibrium payoff must also be strictly positive, as he could earn strictly positive expected profits by offering $k_{2}=k_{h, g}^{I}-\varepsilon$.

If investor 1 makes no offer, then this reveals that $\sigma(q)=b$ (investor 1 cannot randomize between making offers and not making offers when $\sigma(q)=g$ because this would imply zero gross payoff). As a result, $k_{2}>k(q)$ implies that the entrepreneur does not accept investor 2's offer when there is no offer by investor 1 . Now suppose that investor 1 offers $k_{1}>k_{2}$. Because $k_{2}$ is in the relative interior of the support of investor 1's mixed strategy for $\sigma(q)=g$, this occurs with strictly positive probability. Then the entrepreneur's posterior probability that $\sigma(q)=g$ must be uniformly bounded away from 0 for all $k_{1}$ in the support of investor 1's mixed strategy, else offer $k_{1}$ would be rejected by the entrepreneur and investor 1's gross profits would be zero. Clearly, $E\left[k_{1} X \mid \sigma(q)=g\right]>1$ and $E\left[k_{1} X \mid \sigma(q)=b\right] \geq 1$ if $\rho\left(k_{1}\right)<1$. Since $\varepsilon$ can be chosen arbitrarily and the probability that $\sigma(q)=g$ is uniformly bounded from below, it follows that investor 2's expected payoff from $k_{2}=k_{h, g}^{I}-\varepsilon$ is strictly positive.

Indifference implies that both investors must earn positive payoff at $k_{h, g}^{I}$ and $k_{h}^{U}$, respectively. Thus, $k_{h, g}^{I}=k_{h}^{U}$ and these offers must be made with strictly positive probability. This, however, is a contradiction, as investor 2 could strictly increase his payoff by offering $k_{2}$ that is marginally less than $k_{h}^{U}$ because the acceptance probability is increased discontinuously, a contradiction. Thus, $k_{l, g}^{I}=k_{h, g}^{I}=k(q)$. Further, investor 2 must also offer $k_{2}=k(q)$ with sufficiently high probability, else investor 1 would do better to raise his offer, which cannot be supported in equilibrium, as we have just shown. Finally, it follows that investor 1's offer must be accepted when both investors offer the same $k \leq k(q)$, else investor 1 could reduce his offer marginally and fund the project for sure (as any offer $k_{1}<k(q)$ is accepted independently of beliefs). This completes the argument.

Case $3 b$. We can assume that $q>0$, else the result holds trivially. There are two subcases. 
Case 3b, $i$. Suppose that $E[k(q) X \mid \sigma(q)=b] \leq 1$.

We first construct an equilibrium. Recall that $\underline{k}$ is defined by $E[\underline{k} X]=1$. Let $k_{l, g}^{I}=k_{l}^{U}=\underline{k}$ and $k_{h, g}^{I}=k_{h}^{U}=k(q)$. If offers $k>k(q)$ are made by investor 1 (out of equilibrium) then we assume that the entrepreneur believes that $\sigma(q)=b$ with probability 1 . Let $\xi$ be the probability that investor 2 makes an offer, and let $F^{I}$ and $F^{U}$ be the cdfs of informed investor 1's and uninformed investor 2's mixed strategies, respectively. Note that $F^{U}$ is the distribution of $k_{2}$ given that investor 2 makes an offer. Then the respective indifference conditions for the informed and uninformed investor are

$$
\begin{aligned}
0 & =p\left(1-F^{I}(k)\right)(E[k X \mid \sigma(q)=g]-1)+(1-p)(E[k X \mid \sigma(q)=b]-1) \\
E[\underline{k} X \mid \sigma(q)=g]-1 & =\xi\left(1-F^{U}(k)\right)(E[k X \mid \sigma(q)=g]-1)+(1-\xi)(E[k X \mid \sigma(q)=g]-1)
\end{aligned}
$$

Solving the indifference equations for $F^{I}$ and $F^{U}$ yields

$$
\begin{aligned}
F^{I}(k) & =1+\frac{1-p}{p} \frac{E[k X \mid \sigma(q)=b]-1}{E[k X \mid \sigma(q)=g]-1}, \\
F^{U}(k) & =\frac{1}{\xi}\left(1-\frac{E[\underline{k} X \mid \sigma(q)=g]-1}{E[k X \mid \sigma(q)=g]-1}\right) .
\end{aligned}
$$

Finally,

$$
\xi=1-\frac{E[\underline{k} X \mid \sigma(q)=g]-1}{E[k(q) X \mid \sigma(q)=g]-1}
$$

implies that $F^{U}(\bar{k})=1$.

Simple algebra reveals that $0 \leq \xi \leq 1$ and that $F^{I}$ and $F^{U}$ are increasing functions of $k$. The indifference equations immediately reveal that $F^{I}(\underline{k})=F^{U}(\underline{k})=0$. Further, $F^{U}(k(q))=1$ while $F^{I}(k(q))<1$, indicating that offer $k(q)=k_{h, g}^{I}$ is made with probability $1-F^{I}(k)>0$. It is also obvious that no investor can improve by making offers outside $[\underline{k}, k(q)]$. In particular, if the informed investor makes an offer $k>k(q)$, it is rejected because the entrepreneur believes that $\sigma(q)=b$ and hence his payoff would be negative.

It remains to prove that in any equilibrium investor 1's payoff $E[\underline{k} X \mid \sigma(q)=g]-1>0$ when $\sigma(q)=g$ and 0 when $\sigma(q)=b$. Clearly, $k_{l}^{U}=k_{l, g}^{I}$. If $k_{l}^{U}<k_{l, g}^{I}$ then uninformed investor 2's profits would increase with $k_{2}$ with $k^{U} \leq k_{2}<k_{l, g}^{I}$, a contradiction since mixing implies indifference. Similarly, we get a contradiction when $k_{l}^{U}>k_{l, g}^{I}$. So suppose that $k_{l, g}^{I}=k_{l}^{U}<\underline{k}$. Since $E[\underline{k} X]=1$, it follows that $E[\underline{k} X \mid \sigma(q)=b]-1<0$ and therefore $k_{l, g}^{I}$ is not be offered by informed investor 1 if $\sigma(q)=b$. This, however, implies that $k_{l}^{U}$ would be accepted by the entrepreneur with probability 1 when $\sigma(q)=b$ since $k_{l}^{U}<k(q)$, which implies that $E\left[\left(1-k_{l}^{U}\right) X \mid \sigma(q)=b\right]>w$. Thus, $k_{l}^{U}<\underline{k}$ and $E[\underline{k} X]-1=0$ imply that investor 2 loses money, a contradiction. Thus, $k_{l, g}^{I} \geq \underline{k}$.

Next, suppose by way of contradiction that $k_{l, g}^{I}>\underline{k}$. Again, $k_{l}^{U}=k_{l, g}^{I}$. This, however, implies that investor 2's expected payoff is strictly positive, as he can always guarantee himself strictly 
positive profits by offering $k_{2}$ marginally less than $k_{l, g}^{I}$ (since $k_{2}<k(q)$ the offer would be accepted in both states and $k_{2}>\underline{k}$ ensures that $E\left[k_{2} X\right]-1>0$ ). Similarly, it follows that investor 1's profit is strictly positive. Thus, indifference across offers in the support of the mixed strategy implies that profits are strictly positive at $k_{h}^{U}$ and $k_{h, g}^{I}$.

If investor 1 does not offer contracts that are accepted when $\sigma(q)=b$, then strictly positive profits at $k_{h}^{U}$ and $k_{h, g}^{I}$ are only possible if $k_{h}^{U}=k_{h, g}^{I}$ and both are offered with strictly positive probability. However, this implies that investor 2 can strictly improve by offering $k$ that is marginally less than $k_{h, g}^{I}$, because the lower offer discontinuously increases the acceptance probability, a contradiction.

If investor 1 makes offers when $\sigma(q)=b$ then strictly positive profits at $k_{h}^{U}$ and $k_{h, g}^{I}$ imply $k_{h, g}^{I} \leq k_{h}^{U} \leq k_{h, b}^{I}$. By assumption $E[k(q) X \mid \sigma(q)=b] \leq 1$. Thus, $k_{l, b}^{I}>k(q)$, which implies $E\left[\left(1-k_{l, b}^{I}\right) X \mid \sigma(q)=b\right]<w$, i.e., if the entrepreneur learns with probability 1 that $\sigma(q)=b$ then he will not accept the contract. As a consequence, $k_{h, b}^{I} \leq k_{h, g}^{I}$. Thus, $k_{h, g}^{I}=k_{h}^{U}=k_{h, b}^{I}$, implying that both $k_{h}^{U}$ and $k_{h, g}^{I}$ are again offered with strictly positive probability. Thus, we get the same contradiction as in the previous paragraph.

The contradiction implies that $k_{l, g}^{I}=\underline{k}$. This and indifference across the support of the mixing probability, implies that investor 1's expected payoff is $E[\underline{k} X \mid \sigma(q)=g]-1$ when $\sigma(q)=g$.

Now suppose by way of contradiction that investor 1's gross payoff is strictly positive when $\sigma(q)=b$. Then investor 2's equilibrium profits are strictly positive, since investor 2 can get strictly positive profits by offering a $k_{2}$ that is marginally less than $k_{l, b}^{I}$, which is accepted with probability 1 when $\sigma(q)=b$. As above, we can conclude that $k_{h, g}^{I}=k_{h}^{U}=k_{h, b}^{I}$. Because $k_{h}^{U}$ and $k_{h, g}^{I}$ are again offered with strictly positive probability, we get the same contradiction, proving that the informed investor's profits are zero.

Case 3b, ii. Suppose that $E[k(q) X \mid \sigma(q)=b]>1$. Let $\bar{k}$ solve $E[\bar{k} X \mid \sigma(q)=b]=1$. Obviously, $\bar{k}<k(q)$. Now investor 1 offers $\bar{k}$ if $\sigma(q)=b$ and mixes over $[\underline{k}, \bar{k}]$ when $\sigma(q)=g$. When $\bar{k}$ is offered by both investors, investor 1's offer is accepted. The cdf $F^{I}$ is again given by (7), because the indifference equation of investor 2 is again given by (5). The indifference equation of investor 1 is

$$
E[\underline{k} X \mid \sigma(q)=g]-1=\left(1-F^{U}(k)\right)(E[k X \mid \sigma(q)=g]-1)
$$

Thus,

$$
F^{U}(k)=1-\frac{E[\underline{k} X \mid \sigma(q)=g]-1}{E[k X \mid \sigma(q)=g]-1)} .
$$

Note that $1-F^{U}(\bar{k})>0$ and thus uninformed investor 2 offers $k_{2}=\bar{k}$ with strictly positive probability. (5) implies that $F^{I}(\bar{k})=1$. Establishing that this is an equilibrium follows along the lines of Case 3b, i. Note that investor 1's offer must be accepted when the investors make the same offer 
$k_{1}=k_{2}=\bar{k}$; if not, investor 1's payoff would drop at $\bar{k}$, destroying indifference to $k_{1}$ in the support of his mixed strategy.

It remains to characterize investor 1's payoffs in all equilibria of the subgame. We first show that investor 1's expected payoff is $E[\underline{k} X \mid \sigma(q)=g]-1>0$ when $\sigma(q)=g$. The argument used in case $3 \mathrm{~b}, \mathrm{i}$ implies $k_{l}^{U}=k_{l, g}^{I} \geq \underline{k}$. Thus, investor 1's equilibrium payoff is strictly positive. If $k_{l}^{U}=k_{l, g}^{I}>\underline{k}$ we can again conclude that investor 2's payoff is also strictly positive. Thus, $k_{h, g}^{I}$ must be accepted with strictly positive probability, which implies $k_{h, g}^{I} \leq k_{h}^{U}$. If $k_{h, g}^{I}=k_{h}^{U}$ then the fact that both $k_{h, g}^{I}$ and $k_{h}^{U}$ must be offered with strictly positive probability implies a contradiction as above. Thus, $k_{h, g}^{I}<k_{h}^{U}$. This, however, can only be supported in equilibrium if $k_{h, b}^{I}=k_{h}^{U}$. But in order for investor 2's profits to be strictly positive, $k_{h, b}^{I}$ must be offered with strictly positive probability. Again, this gives a contradiction because investor 2 could then increase profits by offering $k$ that is marginally less than $k_{h}^{U}$. Thus, $k_{l}^{U}=k_{l, g}^{I}=\underline{k}$, implying that investor 1's expected profits are $E[\underline{k} X \mid \sigma(q)=g]-1$ when $\sigma(q)=g$.

Now suppose by way of contradiction that the informed investor's payoff is strictly positive when $\sigma(q)=b$. Then profits must be strictly positive at $k_{h, b}^{I}$ and this offer must be accepted with strictly positive probability. However, this implies that investor 2's profits must be positive as well, as he could offer $k$ marginally smaller than $k_{h, b}^{I}$ which would be accepted at least when $\sigma(q)=b$. Thus, both investors would make strictly positive profits, and repeating the above arguments a contradiction follows. Thus, investor 1's profits are 0 when $\sigma(q)=b$.

Case 3c. If $E[X \mid \sigma(q)=g]<1$ then not offering any contract is clearly an equilibrium. Now suppose that $E[X \mid \sigma(q)=g] \geq 1$. Choose $k$ such that $E[k X \mid \sigma(q)=g]=1$. Then both investors offering $k$ and the entrepreneur accepting the contract if and only if the informed investor makes an offer, is an equilibrium. The payoff to both investors is zero. Because $k \geq k(q)$, the entrepreneur does not benefit from accepting $k$ when $\sigma(q)=b$, i.e., when investor 1 does not make an offer.

Suppose by way of contradiction that investor 1 makes strictly positive profits. Then $E[X \mid \sigma(q)=$ $g]>1$ and $k_{l, g}^{I}>k(q)$. Then investor 2 must also make strictly positive profits, else he could offer $k$ with $k(q)<k<k_{l, g}^{I}$. Using the same arguments as above, yields a contradiction.

Proof of Theorem 2. For investor 1 to be willing to acquire information, his gross payoff of the stage 2 subgame must cover $c$. Lemma 1 reveals that this is only true in 3(a) and 3(b). Thus, investor 1's payoff given $q$ is

$$
p E[\min \{k(q), \bar{k}\} X \mid \sigma(q)=g]-c .
$$


$q$ must satisfy $E[X \mid \sigma(q)=g]>1+w$ and $E[X \mid \sigma(q)=b] \geq w$. Clearly, $E[X \mid \sigma(q)=g]>1+w$, otherwise investor 1's payoff including costs $c$ would be strictly negative, and he could improve by not investigating. Further, if $q$ is increased such that $E[X \mid \sigma(q)=b]$ becomes close to $w$ then $k(q)$ converges to 0 and thus, (9) converges to $-c$. As a consequence, if an equilibrium with information acquisition exists, then $k(q)$ exists for any $q$ that is chosen in equilibrium. Thus, investor 1's payoff can be obtained by maximizing (9).

Suppose that at the maximum $k(q)>\bar{k}$. In the model section we assume that $E[X \mid \sigma(1)=b]<$ $w$. Thus, $q<1$. Hence we can increase $q$ marginally to $q^{\prime}$. Then $k\left(q^{\prime}\right)>k$, but $(9)$ is increased since the signal is more accurate. Thus, $k(q) \leq k$. Hence, any equilibrium $k$ and $q$ must solve Problem 1.

Conversely, suppose that $k$ and $q$ solve Problem 1. Then Lemma 1 implies that the strategies are an equilibrium and that there exist equilibria in all subgames.

Proof of Theorem 3. Fix the expected project payoff, $E[X]=p \bar{x}$. Then decreasing $p$ while keeping $E[X]$ fixed increases the variance.

If only constraint 1 of Problem 1 binds, then $k=1-\sqrt{\frac{(1-p) w}{p \bar{x}}}, q=1-\sqrt{\frac{w}{p \bar{x}(1-p)}}$. Substituting this into the objective of Problem 1 and using the fact that $E[X]=p \bar{x}$ yields investor 1's equilibrium gross payoffs,

$$
(1-p) w+E[X]-c-p-2 \sqrt{E[X](1-p) w} .
$$

The second derivative of $(10)$ with respect to $p$ is $\frac{\sqrt{w E[X]}}{2(1-p)^{3 / 2}}>0$, i.e., the payoff is convex in $p$.

If both constraints of Problem 1 bind, then solving the two constraints reveals that investor 1's payoff is

$$
-\frac{(1-p) w}{E[X]-1}+(1-p)-c
$$

which is a linear function of $p$.

Optimization by investor 1 implies that this line is tangent to (10), so that if constraint 2 binds then it binds for all $p$ between the tangency point $p_{X}^{t}$ and $p=1$. Note that if $p=1$ and constraint 2 is slack then $\bar{x}<1+w$, so that financing is unprofitable; and if both constraints bind, then the payoff $(11)$ is $-c$, i.e., informed finance is again not feasible. It follows that in a neighborhood of $p=1$, i.e., when variance is low, that informed finance is not feasible.

Figure 2 depicts the three cases that can arise. The first two panels of Figure 2 consider the case where constraint 2 binds for sufficiently large $p$, and the right panel considers the case where constraint 2 never binds. In all three cases it follows immediately that if only the first constraint binds and the net payoff is non-negative then the convex payoff curve must be decreasing in $p$, 


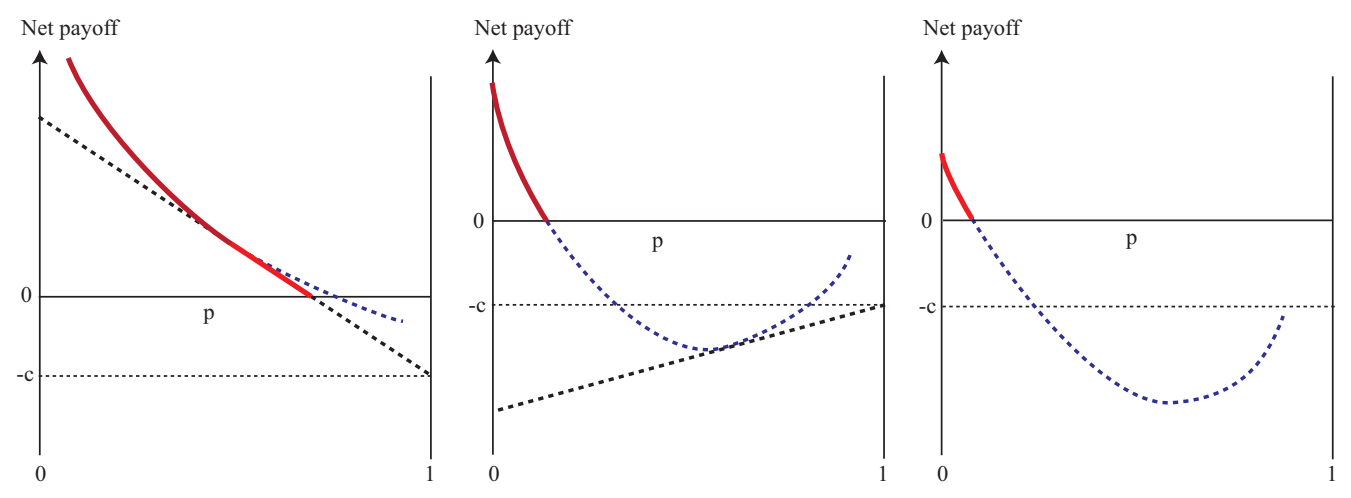

Figure 2: The profit of an informed investor when $E[X]$ is kept fixed

i.e., the payoff is strictly increasing in the variance, whenever informed investment occurs. Finally, if both constraints bind then a non-negative payoff can only occur if the linear payoff curve is decreasing (as the payoff at $p=1$ is $-c$ ). Again, this implies that the payoff on this range is increasing in the variance.

Proof of Lemma 2. The result is standard if $X$ has only a finite number of realizations, so suppose that $f_{j}(x)$ is the density of $X$ given $\sigma(q)=j, j=b, g$. Problem (3) is equivalent to

$$
\max _{\kappa \in \mathfrak{S}} \int x \kappa(x) f_{b}(x) d x \text { s.t. } \int x \kappa(x) f_{g}(x) d x \leq \gamma
$$

Let $\alpha$ be the maximized value of (12). Let $\kappa_{n}, n \in \mathbb{N}$ be a sequence with $\lim _{n \rightarrow \infty} \int x \kappa_{n}(x) f_{b}(x) d x=$ $\alpha$, where $\kappa_{n}$ fulfills the constraint of (12) for all $n \in \mathbb{N}$. Kómlos' Theorem implies that there exists a subsequence $\kappa_{n_{i}}, i \in \mathbb{N}$ and a function $\kappa^{*}$ such that

$$
\lim _{I \rightarrow \infty} \frac{1}{I} \sum_{i=1}^{I} \kappa_{n_{i}}=\kappa^{*}, \text { a.e. }
$$

Convexity and closedness of $\mathfrak{S}$ implies that $\kappa^{*} \in \mathfrak{S}$.

It follows immediately that $\kappa^{*}$ fulfills the constraint of the optimization problem. In particular,

$$
\int x \kappa^{*}(x) f_{g}(x) d x=\lim _{i \rightarrow \infty} \frac{1}{I} \sum_{i=1}^{I} \int x \kappa_{n_{i}} f_{g}(x) d x \leq \gamma,
$$

where the equality follows from Lebesgue's dominated convergence Theorem, and the inequality from the fact that each $\kappa_{n_{i}}$ fulfills the constraint. Thus,

$$
\lim _{I \rightarrow \infty} \int \frac{1}{I} \sum_{i=1}^{I} x \kappa_{n_{i}}(x) f_{b}(x) d x=\lim _{I \rightarrow \infty} \frac{1}{I} \sum_{i=1}^{I} \int x \kappa_{n_{i}}(x) f_{b}(x) d x=\alpha,
$$


as $\lim _{i \rightarrow \infty} \int x \kappa_{n_{i}}(x) f_{b}(x) d x=\alpha$ implies that that averages, given by the right-hand side of (14), converge to $\alpha$ as well. Thus, (13) and Lebesgue's dominated convergence theorem implies that $\kappa^{*}$ solves (12).

Let $\kappa_{\gamma}$ be the solution of (12) for a given $\gamma$. It is immediate that $\int x \kappa_{\gamma}(x) f(x) d x$ is strictly increasing in $\gamma$. It remains to prove continuity. Suppose by way of contradiction that $\int x \kappa_{\gamma}(x) f_{b}(x) d x$ has a discontinuity at $\hat{\gamma}$. Then there exists $\varepsilon>0$ such that for all $\delta>0$ there exists $\gamma<\gamma^{\prime}<\gamma+\delta$ such that

$$
\int x \kappa_{\gamma}(x) f_{b}(x) d x+\varepsilon<\int x \kappa_{\gamma^{\prime}}(x) f_{b}(x) d x .
$$

Let $\kappa(x)=\frac{\gamma}{\gamma^{\prime}} \kappa_{\gamma^{\prime}}(x)$. Then convexity of $\mathfrak{S}$ and the fact that $0 \in \mathfrak{S}$ implies that $\kappa \in \mathfrak{S}$. Note that $\int x \kappa(x) f_{g}(x), d x \leq \gamma$, i.e. $\kappa$ fulfills the constraint of problem (12) for $\gamma$. However, $\int x \kappa(x) f_{b}(x) d x=$ $\frac{\gamma}{\gamma^{\prime}} \int x \kappa(x) f_{b}(x) d x>\int x \kappa_{\gamma}(x) f_{b}(x) d x$ if $\delta$ is sufficiently small. Thus, $\kappa_{\gamma}$ does not solve problem (12) for $\gamma$, a contradiction. Hence continuity follows.

Proof of Theorem 4. Define the equity share $\psi_{s}(\kappa)=\frac{E[\kappa(X) X \mid \sigma(q)=s]}{E[X \mid \sigma(q)=s]}$ for $s=g, b$ that is equivalent to contract $\kappa$ in state $s$. Since all agents are risk neutral, only the equivalent shares matter for calculating payoffs.

We start by analyzing the subgames starting at stage 2, i.e., where $q$ has already been chosen. Claim 1: Let $\mathfrak{K}_{g}$ be the support of contracts offered by informed investor 1 in state $g$. Then

1. $\kappa \in \mathfrak{K}_{g}$ for any contract that is offered by investor 1 and accepted with positive probability in state $b$.

2. If $\sigma(q)=b$, then the probability that investor 1 offers a contract $\kappa \in \mathfrak{K}_{g}$ is strictly less than one.

3. $\max _{\kappa \in \mathfrak{K}_{g}} E[\kappa(X) X \mid \sigma(q)=b] \leq 1$ : investor 1 cannot earn strictly positive gross profits by offering a contract $\kappa \in \mathfrak{K}_{g}$ in state $b$.

Suppose by way of contradiction contract $\kappa \notin \mathfrak{K}_{g}$ is offered in equilibrium. Then in equilibrium the entrepreneur must assign probability 1 to $\sigma(q)=b$. Thus, $E[X \mid \sigma(q)=b] \leq E[X]<1+w$ implies that either the entrepreneur or investor 1 loses money, a contradiction of optimizing behavior.

Next, suppose that investor 1 always offers a contract $\kappa \in \mathfrak{K}_{g}$ when $\sigma(q)=b$. Let $\mathfrak{K}_{b}$ be the support of the contracts offered when $\sigma(q)=b$. Then the first part of claim 1 implies that $\mathfrak{K}_{b} \subset \mathfrak{K}_{g}$. Thus, there exists a contract $\kappa$ such that the entrepreneur believes that $\sigma(q)=g$ has occurred with 
probability $\rho(\kappa)<P(\{\sigma(q)=g\})$. Further, $E[(1-\kappa(X)) X \mid \sigma(q)=b]<w \leq E[(1-\kappa(X)) X \mid \sigma(q)=$ $g]$, where the first inequality holds because investor 1's gross payoff $E[\kappa(X) X \mid \sigma(q)=b]-1 \geq 0$ (else he would not offer investment) and $E[X]<1+w$. Thus,

$$
\rho(\kappa) E[(1-\kappa(X)) X \mid \sigma(q)=g]+(1-\rho(\kappa)) E[(1-\kappa(X)) X \mid \sigma(q)=b] \leq E[(1-\kappa(X)) X] .
$$

Since $E[\kappa(X)) X \mid \sigma(q)=s] \geq 1$ for $s=g, b$, the fact that $E[X]<1+w$ implies $E[(1-\kappa(X)) X]<w$. This and (16) imply that the entrepreneur's expected payoff from accepting contract $\kappa$ is strictly less than $w$, a contradiction.

The third statement of the claim now follows immediately from the second statement.

The remaining argument follows that of Lemma 1 with modifications. Note, that Lemma 1 immediately extends if we have many states but restrict contracts to equity shares. Thus, if we show that the multidimensional contract design problem is equivalent to the one-dimensional problem of choosing an equity share then Lemma 1 can be applied. Also, in contrast to a two state setting, the equilibrium contract of uninformed investor 2 is not unique, but the payoffs are.

Case 1.: No change.

Case 2.: The existence part is the same. In particular, since investor 1 does not offer a contract when $\sigma(q)=b$, the state is revealed to all parties, and given contract offers $\kappa^{i}, i=1,2$ by the two investors, the entrepreneur will choose the contract that corresponds to the lower share in the good state, i.e., for which $\psi_{g}\left(\kappa^{i}\right)$ is less. All profits are competed away.

Conversely, first suppose that investor 1 does not offer a contract when $\sigma(q)=b$. Then since only the equivalent shares $\psi_{g}\left(\kappa^{i}\right)$ matter, the problem is one-dimensional and hence equivalent to that in Lemma 1. Now suppose that investor 1 offers a contract in state $\sigma(q)=b$ with strictly positive probability. Again, assume by way of contradiction that investor 1's gross payoff is strictly positive. Let $\mathfrak{K}_{b}$ be the support of offers when $\sigma(q)=b$. If $\kappa \notin \mathfrak{K}_{g}$ then the state is revealed and the argument above extends. Accordingly, suppose that $\kappa \in \mathfrak{K}_{g}$. Then $\kappa$ must be accepted with strictly positive probability, else investor 1's gross payoff when $\sigma(q)=g$ would be zero when $\kappa$ is offered. This, in turn, would imply that investor 1's payoff is always zero when $\sigma(q)=g$, which would complete the proof. Let $k_{h, g}^{I}=\sup \psi_{g}\left(\mathfrak{K}_{g}\right)$. If $\psi_{g}(\kappa)<k_{h, g}^{I}$ then investor 2 could get a strictly positive payoff from offering $\kappa$. Thus, investor 2's equilibrium payoff would have to be positive, and we get a contradition as in Lemma 1. Thus, suppose that $\psi_{g}(\kappa)=k_{h, g}^{I}$. Then investor 2 could make strictly positive profits by offering a contract $\lambda \kappa$, where $\lambda<1$ but sufficiently close to 1 , and a contradiction again obtains.

Cases 3. If $E[X \mid \sigma(q)=b] \geq w$ then Lemma 2 implies that there exists a contract $\hat{\kappa}(x)$ such that 
(i) $E[(1-\hat{\kappa}(X)) X \mid \sigma(q)=b]=w$, and (ii) there does not exist a contract $\kappa^{\prime}(\cdot)$ with $\psi_{g}\left(\kappa^{\prime}\right) \leq \psi_{g}(\hat{\kappa})$ and $E\left[\left(1-\kappa^{\prime}(X)\right) X \mid \sigma(q)=b\right]<w$.

Case 3a. Suppose that $E[\hat{\kappa}(X) X] \leq 1$ and $E[k(q) X \mid \sigma(q)=g]>1$ where $k(q)=\psi_{g}(\kappa)$. The argument of Lemma 1 implies that the following is an equilibrium: investor 1 offers $k(q)$ if and only if $\sigma(q)=g$, and investor 2 offers $\hat{\kappa}(\cdot)$, which is not accepted in equilibrium. It remains to prove that it is not optimal for investor 1 to offer a contract when $\sigma(q)=b$. Let $\kappa$ be a contract offered when $\sigma(q)=b$. If $E[(1-\kappa(X)) X \mid \sigma(q)=b]<w$ then $\kappa$ will not be accepted, if we assign to the entrepreneur the belief that $\sigma(q)=b$. Thus, the deviation does not increase investor 1's payoff. If, instead, $E[(1-\kappa(X)) X \mid \sigma(q)=b] \geq w$ then the contract is acceptable to the entrepreneur even if $\sigma(q)=b$. However, $E[X]<1+w$ implies $E[\kappa(X) X \mid \sigma(q)=b]-1<0$, i.e., investor 1 loses money and the deviation makes him worse off.

We now characterize all equilibria of this subgame. Again we can conclude that any contract $\kappa$ with $\psi_{g}(\kappa)<k(q)$ is accepted by the entrepreneur independently of beliefs, since $E[(1-$ $\kappa(X)) X \mid \sigma(q)=s]>w$ for $s=g, b$ by the definition of $k(q)$, and because $\psi_{g}(\kappa)<k(q)$. Thus, $E[k(q) X] \leq 1$ implies that investor 1 never offers such a contract when $\sigma(q)=b$.

Let $k_{l}^{U}=\min \psi_{g}\left(\mathfrak{K}^{U}\right)$, where $\mathfrak{K}^{U}$ is the support of investor 2's possibly mixed strategy. As in Lemma 1 we can prove that $k^{U} \geq k(q)$. In particular, if $k_{l}^{U}<k(q)$ then the definition of $k(q)$ and the fact that $E[k(q) X] \leq 1$ imply that the contract is accepted by the entrepreneur at least when $\sigma(q)=b$ (because informed investor 1 never makes a competing offer when $\sigma(q)=b$ ), resulting in a negative payoff to investor 2 .

Let $k_{l}^{I}=\min \psi_{g}\left(\mathfrak{K}^{I}\right)$ and $k_{h}^{I}=\max \psi_{g}\left(\mathfrak{K}^{I}\right)$, where $\mathfrak{K}^{I}$ is the support of informed investor 1 's strategy. Since $k^{U} \geq k(q)$, it follows that $k_{l}^{I} \geq k(q)$. We next prove that $k_{h}^{I}=k(q)$. Suppose by way of contradiction that $k_{h}^{I}>k(q)$. Then we can conclude as in Lemma 1 that investor 2's payoff is also strictly positive. Thus, both investor 1 and investor 2 must make strictly positive profits at $k_{h}^{I}$ and $k_{h}^{U}$, and the standard contradiction obtains. It therefore follows that $k^{U}=k_{h}^{I}=k_{l}^{I}$. Moreover, the contract offered by uninformed investor 2 must maximize $\psi_{b}(\kappa)$ keeping $\psi_{g}(\kappa)$ fixed, i.e., must satisfy (3).

Cases 3b. Suppose that $E[\hat{\kappa}(X) X]>1$. Note that there is no further division into subcases (i) and (ii), because $E[\hat{\kappa}(q) X \mid \sigma(q)=b]<1$ as $E[(1-\hat{\kappa}(X) X \mid \sigma(q)=b] \geq w$ and $E[X]<1+w$.

To construct an equilibrium, define for each $k$ the contract $\kappa_{k}$ that solves max $E[(1-\kappa(X)) X \mid \sigma(q)=$ $b]$ subject to $\psi_{g}(\kappa)=k$. Choose $\underline{k}$ such that $E\left[\kappa_{\underline{k}}(X) X\right]=1$. Let $\mathfrak{K}=\left\{\kappa_{k} \mid k \in[\underline{k}, k(q)\}\right.$. We can therefore describe the mixed strategies of the uninformed and the informed party, respectively, as 
cdfs $F^{U}$ and $F^{I}$ on $[\underline{k}, k(q)]$. Then (6) of Lemma 1 again describes the indifference condition of the informed investor, and $F^{U}$ is again given by (8). The indifference curve of investor 2 is

$$
0=p\left(1-F^{I}(k)\right)(E[k X \mid \sigma(q)=g]-1)+(1-p)\left(E\left[\kappa_{k}(X) X \mid \sigma(q)=b\right]-1\right) .
$$

It follows immediately that $F^{I}(k)$ is increasing and that $F^{I}(\underline{k})=0$. Moreover, since $E[\hat{\kappa}(X) X]>1$ and $\hat{\kappa}=\kappa_{k(q)}$ it follows that $F^{I}(k(q))<1$, i.e., $k(q)$ is offered with strictly positive probability.

The proof that all equilibria are payoff equivalent to the equilibrium just constructed is almost identical to Lemma 1. For informed investor 1, we can identify the set of contracts $\mathfrak{K}_{g}^{I}$ offered in

state $\sigma(q)=g$ by the set of equivalent shares $\psi_{g}\left(\mathfrak{K}_{g}^{I}\right)$. As shown at the beginning, offers by investor 1 when $\sigma(q)=b$ yield zero gross payoff and therefore do not contribute to his payoffs. Finally, uninformed investor 2 must always choose a contract that solves $\max \psi_{b}(\kappa)$ subject to $\psi_{g}(\kappa)=k$, where $k$ is a fixed share. Otherwise, if $\kappa$ does not solve this optimization problem, the uninformed investor could strictly increase his payoff by offering $\kappa^{\prime}$ with $\psi_{g}\left(\kappa^{\prime}\right)=\psi_{b}(\kappa)$ but $\psi_{b}\left(\kappa^{\prime}\right)>\psi_{b}(\kappa)$, because informed investor 1 does not compete in state $b$.

The remainder of the proof follows along the lines of Theorem 2. However, note that if Problem 2 does not have a solution then this implies that the game does not have an equilibrium.

Proof of Theorem 5. Note that (4) implies

$$
\lim _{x \rightarrow y} \frac{d}{d x}\left[\frac{F_{g}(x)-F_{g}(y)}{F_{b}(x)-F_{b}(y)}\right]>0 .
$$

In particular,

$$
\frac{d}{d x}\left[\frac{F_{g}(x)-F_{g}(y)}{F_{b}(x)-F_{b}(y)}\right]=\frac{f_{g}(x)\left(F_{b}(x)-F_{b}(y)\right)-\left(F_{g}(x)-F_{g}(y)\right) f_{b}(x)}{\left(F_{g}(x)-F_{g}(y)\right)^{2}} .
$$

To determine the limit in (19), we apply L'Hôspital's rule. The first derivatives of the numerator and the denominator evaluated at $y=x$ are both zero. Taking the second derivatives and evaluating them at $y=x$ yields

$$
\lim _{x \rightarrow y} \frac{d}{d x}\left[\frac{F_{g}(x)-F_{g}(y)}{F_{b}(x)-F_{b}(y)}\right]=\frac{f_{g}^{\prime}(x) f_{b}(x)-f_{g}(x) f_{b}^{\prime}(x)}{2 f_{b}^{2}(x)},
$$

which is strictly greater than zero if (4) holds. Thus, (18) holds.

Next, note that (18) implies that for any sufficiently small interval $I=[\underline{y}, \bar{y}]$, and for all $x \in I$ the following holds:

$$
\frac{F_{g}(x)-F_{g}(\underline{y})}{F_{g}(\bar{y})-F_{g}(\underline{y})}<\frac{F_{b}(x)-F_{b}(\underline{y})}{F_{b}(\bar{y})-F_{b}(\underline{y})}
$$


Hence, the conditional distribution $F_{g}(x \mid I)$ first order stochastically dominates $F_{b}(x \mid I)$.

Let $h^{*}(\cdot)$ solve

$$
\max _{h(\cdot)} \int h(x) d F_{b}(x) \text { s.t. }
$$

1. $\int h(x) d F_{g}(x)=\bar{k}$;

2. $\frac{h(x)}{x}=\kappa(x) \in \mathfrak{S}$.

Suppose by way of contradiction that $h^{*}$ is not simple debt. Then there exists a sufficiently small interval $I=[\underline{y}, \bar{y}]$ such that: (i) $h^{*}$ is not constant a.e on $I$, and (ii) $h^{*}(\bar{y})<\underline{y}$. Define $\lambda=\int_{I} h^{*}(x) d F_{g}(x \mid I)$. Since $h^{*}$ is montonone and not constant on $I$ and $F_{g}(x \mid I)$ first order stochastically dominates $F_{b}(x \mid I)$,

$$
\lambda=\int_{I} h^{*}(x) d F_{g}(x \mid I)>\int_{I} h^{*}(x) d F_{b}\left(x \mid I_{\delta}\right) .
$$

Let

$$
\hat{h}(x)= \begin{cases}\lambda & \text { if } x \in I ; \\ h^{*}(x) & \text { otherwise. }\end{cases}
$$

Then $\int \hat{h}(x) d F_{g}(x)=\lambda\left(F_{g}(\bar{y})-F_{g}(\underline{y})\right)+\int_{I^{c}} h^{*}(x) d F_{g}(x)=\int_{I} h^{*}(x) d F_{g}(x)+\int_{I^{c}} h^{*}(x) d F_{g}(x)=\bar{k}$. Thus, $\hat{h}$ satisfies constraint 1 . Further, $0 \leq \hat{h}(x) \leq x$ for all $x \operatorname{since} h(\bar{y})<\underline{y}$. Thus, $\kappa(x)=\hat{h}(x) / x \in$ $[0,1]$. Finally, $\hat{h}(x)$ is non decreasing. Next, $(21)$ implies

$$
\begin{aligned}
\int \hat{h}(x) d F_{b}(x) & =\lambda\left(F_{b}(\bar{y})-F_{b}(\underline{y})\right)+\int_{I^{c}} h^{*}(x) d F_{b}(x) \\
& >\int_{I} h^{*}(x) d F_{b}(x \mid I)\left(F_{b}(\bar{y})-F_{b}(\underline{y})\right)+\int_{I^{c}} h^{*}(x) d F_{b}(x)=\int h^{*}(x) d F_{g}(x) .
\end{aligned}
$$

Thus, $\hat{h}$ satisfies the constraints and raises the value of the objective, a contradiction to the optimality of $h^{*}$.

Proof of Theorem 6. In text.

Proof of Theorem 7. We first analyze the subgames starting at the point where the entrepreneur has accepted the ex-ante contract. It is sufficient to characterize equilibria of the subgame where the gross payoff to investor 1 is strictly positive. We first claim that in all such equilibria investor 1 must extent funding if and only if $\sigma(q)=g$. Suppose by way of contradiction that investor 1 offers funding also when $\sigma(q)=b$ and that he reveals the signal to the investor by offering a state contingent contract $\kappa_{g}^{I} \neq \kappa_{b}^{U}$. Then $E\left[\kappa_{s}^{I}(X) X \mid \sigma(q)=s\right] \geq E\left[\kappa_{s^{\prime}}^{I}(X) X \mid \sigma(q)=s^{\prime}\right]$, for $s \neq s^{\prime}$. 
Furthermore, $E\left[\kappa_{s}^{I}(X) X \mid \sigma(q)=s\right] \geq 1$, for $s=g, b$, where at least one inequality is strict, else investor 1 would lose from investigation.

First, if funding is never offered then investor 1's gross profit is obviously zero. If funding is not contingent on the signal then $E\left[\kappa^{I}(X) X\right]-1>0$. Thus, investor 2 could undercut marginally, i.e., offer $\kappa^{U}=t \kappa^{I}$ for $t=1-\epsilon$ for small $\epsilon>0$, and be accepted. ${ }^{9}$ Since investor 2 offers his contract after investor 1 the only equilibrium in the subgame is where investor 2 also offers $\kappa^{I}$, and his offer is always accepted. Thus, investor 1's gross profits would be zero.

Next suppose that $E[X \mid \sigma(q)=b]<w$. Then $E\left[\left(1-\kappa^{U}(X)\right) X \mid \sigma(q)=b\right]$ for all feasible contracts $\kappa^{U}$. As a consequence, the entrepreneur never accepts a contract from investor 2 if investor 1 does not offer funding. Investor 2 can therefore undercut $\kappa^{I}$ and make strictly positive profits. Again, in the only equilibrium in the subgame, investor 2 offers the same contract as investor 1 , and his offer is accepted if and only if $\sigma(q)=b$. Thus, investor 1's gross profits would be zero.

We can therefore assume that $E[X \mid \sigma(q)=b] \geq w$. Thus, Lemma 2 implies that there exists $k(q)$ and $\kappa^{U}$ such that $\kappa^{U}$ solves Problem 3 given $k=k(q)$ and constraint 1 of Problem 2 holds, i.e., $E\left[\left(1-\kappa^{U}(X)\right) X \mid \sigma(q)=b\right]=w$. Let $\psi_{s}(\kappa)=\frac{E[\kappa(X) X \mid \sigma(q)=s]}{E[X \mid \sigma(q)=s]}$ for $s=g, b$. Suppose that $\psi_{g}\left(\kappa^{I}\right)>k(q)$. Let $\tilde{\kappa}^{U}$ solve Problem 3 given $k=\psi_{g}\left(\kappa^{I}\right)$. Then $E\left[\left(1-\tilde{\kappa}^{U}(X)\right) X \mid \sigma(q)=b\right]<w$. As a consequence, when $\sigma(q)=b$ the entrepreneur rejects any contract offered by investor 2 with $t \tilde{\kappa}^{U}$, where $t=1-\epsilon$, for small $\epsilon>0$. The argument of the previous paragraph therefore implies again that investor 1's profits are zero.

We can therefore assume that $\psi_{g}\left(\kappa^{I}\right) \leq k(q)$. Let $\tilde{\kappa}^{U}$ be the contract that solves Problem 3 for $k=\psi_{g}\left(\kappa^{I}\right)$. If $E\left[\tilde{\kappa}^{U}(X) X\right]-1>0$ then investor 2 can again undercut, leaving investor 1 with zero gross profits. Thus, $E\left[\tilde{\kappa}^{U}(X) X\right]-1 \leq 0$. Given this contract, investor 2 will abstain from making an offer when $\psi_{g}\left(\kappa^{I}\right)<k(q)$, since his offer would be accepted by the entrepreneur when $\sigma(q)=b$ and investor 2 would lose money. Finally, if $\psi_{g}\left(\kappa^{I}\right)=k(q)$ then in equilibrium investor 2 can either offer $\kappa^{U}$ or make no offer.

The ex-ante contract that maximizes investor 1's payoff therefore solves Problem 2 except that equality constraint 1 is replaced by $E\left[\left(1-\kappa^{U}(X)\right) X \mid \sigma(q)=b\right] \geq w$. To conclude the proof it is therefore sufficient to show that this inequality constraint binds at an optimum.

Suppose by way of contradiction that $E\left[\left(1-\hat{\kappa}^{U}(X)\right) X \mid \sigma(\hat{q})=b\right]>w$ at a solution $\hat{\kappa}^{U}, \hat{q}, \hat{k}$. Let $\tilde{k}$ be marginally greater the $\hat{k}$. Lemma 2 implies that $\kappa^{U}$ that solves Problem 3 for $k=\tilde{k}$ satisfies $E\left[\left(1-\tilde{\kappa}^{U}(X)\right) X \mid \sigma(\hat{q})=b\right]>w$. Thus, $\tilde{\kappa}^{U}, \hat{q}, \tilde{k}$ satisfy all constraints of the modified optimization problem, however, the objective is increased, a contradiction.

\footnotetext{
${ }^{9}$ Note that $0 \in \mathfrak{S}$ and convexity imply that $\kappa^{U}=t \kappa^{I} \in \mathfrak{S}$.
} 


\section{References}

[1] F. Allen and D. Gale. Measurement distortion and missing contingencies in optimal contracts. Economic Theory, 2:1-26, 1992.

[2] D. Baron and R. Myerson. Regulating a monopolist with unknown costs. Econometrica, 50:911-930, 1982.

[3] T. Broecker. Credit-worthiness tests and interbank competition. Econometrica, 58:429-452, 1990.

[4] R. Engelbrecht-Wiggans, P. R. Milgrom, and R. J. Weber. Competitive bidding and proprietary information. Journal of Mathematical Economics, 11:161-169, 1983.

[5] D. Gale and M. Hellwig. Incentive-compatible debt contracts: The one period problem. Review of Economic Studies, 52:647-663, 1985.

[6] S. Grossman and J. E. Stiglitz. On the impossibility of informationally efficient markets. American Economic Review, 66:393-408, 1980.

[7] M. Hellwig. On the aggregation of information in competitive markets. Journal of Economic Theory, 22:279-312, 1980 .

[8] F. K. Jacques Cremer and J.-C. Rochet. Contracts and productive information gathering. Games and Economic Behavior, 25:174-193, 1998.

[9] S. Krasa and A. Villamil. Optimal contracts when enforcement is a decision variable. Econometrica, 68:119-134, 2000.

[10] J. Lacker and J. Weinberg. Optimal contracts under costly state falsification. Journal of Political Economy, 97:1345-1363, 1989.

[11] R. Townsend. Optimal contracts and competitive markets with costly state verification. Journal of Economic Theory, 2:1-29, 1979.

[12] C. Wang and S. D. Williamson. Debt contracts and financial intermediation with costly screening. Canadian Journal of Economics, 31:573-595, 1998.

[13] S. Williamson. Costly monitoring, financial intermediation, and equilibrium credit rationing. Journal of Monetary Economics, 18:159-179, 1986. 